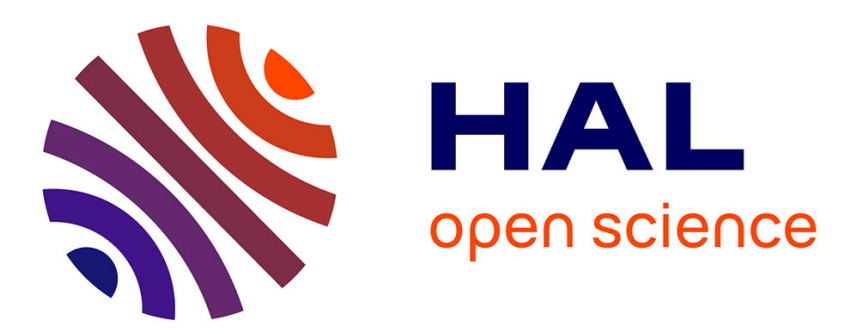

\title{
Expérimentation de la négociation interindividuelle entre cadres et ouvriers dans le contexte socio-politique chinois actuel
}

\author{
Ziyuan Tang, Jean Ruffier
}

\section{- To cite this version:}

Ziyuan Tang, Jean Ruffier. Expérimentation de la négociation interindividuelle entre cadres et ouvriers dans le contexte socio-politique chinois actuel. Revue Recherche en Sciences de Gestion-Management Sciences-Ciencias de Gestión, 2021, 142, pp.309-332. halshs-03413492

HAL Id: halshs-03413492

https://shs.hal.science/halshs-03413492

Submitted on 3 Nov 2021

HAL is a multi-disciplinary open access archive for the deposit and dissemination of scientific research documents, whether they are published or not. The documents may come from teaching and research institutions in France or abroad, or from public or private research centers.
L'archive ouverte pluridisciplinaire HAL, est destinée au dépôt et à la diffusion de documents scientifiques de niveau recherche, publiés ou non, émanant des établissements d'enseignement et de recherche français ou étrangers, des laboratoires publics ou privés. 
revue Recherche en Sciences de Gestion-Management Sciences-Ciencias de Gestión, n¹42, p.

\title{
Expérimentation de la négociation interindividuelle entre cadres et ouvriers dans le contexte socio-politique chinois actuel
}

\author{
Ziyuan Tang \\ Attaché temporaire d'enseignement \& de recherche \\ ISEOR, Magellan \\ Université Jean Moulin Lyon 3 \\ (France) \\ Jean Ruffier \\ DR CNRS, Professeur émérite, \\ ISEOR, Magellan \\ Université Jean Moulin Lyon 3 \\ (France)
}

Cet article évalue une expérience de négociation interindividuelle dans une usine chinoise. Le contexte politique actuel, ainsi que les traditions locales de management, interdisent pratiquement de donner un pouvoir de négociation aux salariés. La méthode choisie a consisté à prendre l'intervenant comme un médiateur qui remplace la négociation directe par des échanges sur les types de changements à introduire et les sanctions/récompenses afférentes. L'expérience s'est faite sur un groupe expérimental, contrasté par un groupe témoin. L'évaluation positive, tant au plan économique que social, montre la faisabilité et l'acceptabilité de cette démarche en Chine.

Mots-clés : Management socio-économique - Motivation au travail Négociation interindividuelle - Amélioration continue - Entreprises chinoises. 
This paper evaluates an experience of inter-individual negotiation in a Chinese factory. It appears difficult, in the current political context, and managerial habits, to give bargaining power to employees. The method chosen was to take the speaker as a mediator who replaces direct negotiation with exchanges on the type of changes to be introduced and the associated sanctions / rewards. The experiment was carried out on an experimental group, contrasted by a control group. The positive evaluation, both economically and socially, shows the feasibility and acceptability of this approach in China.

Key-words: Socio-Economic Approach to Management - Work motivation - Inter-individual negotiation - Continuous improvement Chinese companies.

Este artículo evalúa una experiencia de negociación interindividual en una fábrica china. El contexto político actual, así como las tradiciones de gestión local, prohibe otorgar a los empleados poder de negociación. El método elegido consistió en tomar al consultor como mediador que reemplaza la negociación directa con intercambios sobre el tipo de cambios a introducir y las sanciones / recompensas asociadas. El experimento se llevó a cabo en un grupo experimental, contrastado por un grupo de control. La evaluación positiva, tanto económica como social, muestra la viabilidad y aceptabilidad de este enfoque en China.

Palabras clave: Gestión socioeconómica - Motivación en el trabajo Negociación interindividual - Mejora continua - Empresas chinas.

\section{Introduction}

Le paradigme de la soumission constitue un courant dominant de gestion au sein des entreprises chinoises. Au début de la réforme et de l'ouverture de la Chine (1978), les entreprises chinoises se sont appuyées sur l'approche taylorienne pour intégrer rapidement des salariés chinois de zone rurale, généralement peu qualifiés, dans l'industrie moderne (Ruffier, 2006). Aujourd'hui, le LEAN est considéré comme étant une des approches managériales les plus efficaces pour améliorer la performance manufacturière (Womack et 
Jones, 1996). Cette doctrine est donc largement développée au sein des entreprises chinoises. Toutefois, les résultats obtenus semblent inférieurs à ce que l'on observe au Japon ou dans les pays occidentaux : le LEAN provoque bien une amélioration des résultats mais cette dernière n'est pas durable et les résultats plafonnent (Zhao et Rigas, 2014). Les causes sont principalement humaines : les salariés semblent rarement favorables aux améliorations imposées par la direction et ils ne désirent apparemment pas effectuer des améliorations à leur niveau. Comparés à ceux de la génération antérieure, les salariés chinois de la génération actuelle sont mieux formés et plus qualifiés. Ils expriment plus facilement des besoins personnalisés et sont plus exigeants en matière d'organisation et de conditions du travail. Ils n'ont plus le niveau de docilité ${ }^{1}$ que supposent les méthodes chinoises de management, et cela se traduit par une faible efficacité de ces méthodes. Tout se passe comme si les managers chinois s'efforçaient de moderniser leur management sans remettre en cause le paradigme de la soumission, comme l'ont pourtant fait tous les courants occidentaux ou japonais dont ils s'inspirent. En occident et au Japon, il est normal de considérer les salariés comme des personnes avec lesquelles il faut négocier les changements à introduire. Ce n'est pas le cas en Chine d'aujourd'hui, la plupart des cadres évitent de trop discuter avec les salariés, soit qu'ils se réfèrent à une soi-disant tradition confucéenne qui considère que les subordonnés doivent obéir sans discuter, soit qu'ils pensent que c'est au Parti Communiste et au syndicat de définir ce dont les salariés ont besoin. Dans ce contexte, notre recherche a été menée avec pour objectif de prouver que, même en Chine, une démarche de négociation, plus que d'imposition, pouvait s'avérer plus performante que celles généralement appliquées dans ce pays.

\section{1. - Revue de la littérature}

\footnotetext{
${ }^{1}$ Cette docilité a souvent été avancée par les dirigeants occidentaux pour expliquer leur décision d'implanter des établissements industriels en Chine (Ruffier 2006). Sur l'évolution de la classe ouvrière chinoise, voir Roulleau-Berger (2008), et notamment les textes de LI Peilin qui y sont inclus dans l'ouvrage cité.
} 


\subsection{Virus TFW et paradigme de la soumission}

Le virus taylorisme-fayolisme-weberisme (virus TFW) est un concept, créé par Savall et Zardet, à partir d'une critique des principes proposés par Taylor, Fayol et Weber (Savall 1975 ; Savall et Zardet, 2006). La métaphore du virus TFW «se réfère à la survivance des principes de l'École Classique de l'Organisation, proposée par Taylor (1911), Fayol (1916) et Weber (1924) qui, au demeurant, ont contribué à leur époque au progrès économique et social. Il est toutefois regrettable qu'un siècle plus tard, les théoriciens, les experts et les praticiens continuent à propager trois principes devenus obsolètes : la division maximale du travail, la dichotomie entre conception, décision et réalisation des activités, ainsi que la dépersonnalisation des postes de travail, des organigrammes, des processus, des méthodes et des règles (Savall 1974, 1975, 1979 ; Lussato, 1972 ; Montmollin, 1981). Ces facteurs ne contribuent plus à la performance globale durable, compte tenu des évolutions des comportements, des compétences, de l'environnement social et politique national et international (Savall, 1974, 1981, 2020) » (Savall, Péron, Zardet et Bonnet, 2015).

Le maintien du paradigme de la soumission pousse à une application anachronique des principes proposés par Taylor, Fayol et Weber. Il repose sur une croyance en l'efficacité du système de subordination. Pour Savall et Zardet, le paradigme de la soumission considère que "certains hommes sont subordonnés à d'autres dans l'entreprise, et qu'ils doivent donc, ipso facto, obéir aux ordres et instructions de ceux qui les dirigent. Ce paradigme débouche sur la dépersonnalisation dans la relation de subordination, fonde le rationalisme, la suprématie de la hiérarchie et de la règle qui s'impose sui generis et, in fine, une certaine forme d'élitisme » (Savall et Zardet, 2005). Cependant, ces principes d'hyperspécialisation du travail, de séparation entre les fonctions de conception d'exécution, ainsi que de dépersonnalisation des processus des méthodes de travail affectent la performance socio-économique d'une organisation, et, dans le contexte actuel, deviennent un véritable obstacle au développement durable de la performance globale des entreprises (Savall et Zardet 2015).

\subsection{Motivation au travail}

Le terme motivation définit ce qui détermine ou non la mise en œuvre d'un effort par les salariés. Depuis les années 1930, les théoriciens et les praticiens ne cessent de rechercher la meilleure façon 
de motiver les salariés. Suivant la taxinomie de Kanfer (1990), les théories motivationnelles sont regroupées en trois grandes catégories :

(1) Les théories des besoins-mobiles-valeurs supposent que les actions humaines sont guidées par la satisfaction de besoins multidimensionnels qui sont le reflet de traits personnels (Maslow, 1943 ; Herzberg, 1986). Les salariés peuvent être motivés par des éléments tels que la récompense, la communication, l'autonomie, le feed-back ou encore, le contenu du travail (Deci et Ryan, 1985 ; Hackman et Oldham, 1980). Certains auteurs donnent une place majeure au sentiment de justice ou d'injustice des récompenses attribuées dans les facteurs de motivation (Adams, 1963 ; Greenberg, 1987).

(2) Dans les théories du choix cognitif, la théorie des attentes propose un mécanisme par lequel la performance est fonction de la motivation des salariés (Charles-Pauvers et al, 2006). Ce mécanisme peut être résumé en trois étapes : (a) le salarié perçoit qu'il est en mesure d'atteindre son niveau de performance, c'est donc une attente effortperformance (expectation); (b) il anticipe une récompense (intrinsèque et/ou extrinsèque) qui lui sera attribuée lorsque cette performance sera atteinte, c'est donc une attente performance-résultat (instrumentalité) ; (c) finalement cette récompense sera jugée par lui comme désirable ou non (valence) ce qui conditionne son effort à venir (Vroom, 1964 ; Forest, 2008).

(3) Parmi les théories de l'autorégulation-métacognition, la théorie de la fixation des objectifs postule que la motivation du salarié repose sur le fait qu'il s'efforce de réaliser des objectifs difficiles et spécifiques (Roussel 2000). Les salariés trouveraient une satisfaction à atteindre des objectifs ambitieux. Un niveau d'engagement élevé n'est donc pas en soi surprenant : il constitue une autoréalisation personnelle avant que d'être une performance d'une méthode managériale (Locke et Latham, 1984).

Face à ces avancées théoriques, les faiblesses du paradigme de la soumission à l'égard de la motivation des salariés sont évidentes : ce paradigme réduit le salarié à une sorte d'homo economicus, au lieu d'en faire un être social.

\subsection{La négociation interindividuelle issue du management socio- économique}


Le management socio-économique, créé et expérimenté par Henri Savall et développé avec Véronique Zardet et l'équipe de l'ISEOR, est un management global de l'entreprise qui repositionne l'humain au cœur de l'organisation. Il est fondé sur le développement $\mathrm{du}$ potentiel humain comme levier primordial d'amélioration des performances économiques. $\mathrm{Au}$ contraire du paradigme de la soumission, le management socio-économique constate une désobéissance généralisée des salariés. Il propose des méthodes et des principes organisationnels visant une obtention durable conjointe de la performance sociale et de la performance économique (Savall et Zardet, 2014).

Élément indispensable du management socio-économique, la négociation interindividuelle consiste en une concertation entre un salarié et son supérieur hiérarchique direct que nous appellerons négociation interindividuelle. Elle sert à l'établissement de contrats d'activités périodiquement négociables $(\mathrm{CAPN})^{2}$, lesquels impliquent :

- une contrepartie financière supplémentaire pour rémunérer l'atteinte des objectifs négociés ;

- une mise à plat des comportements réels ;

- une négociation avec le personnel des changements de comportements susceptibles de produire des améliorations quantifiables, ainsi que des récompenses en cas d'atteinte de ces améliorations. (Savall et Zardet, 1987)

Le CAPN contribue à réduire les tensions sociales en augmentant la zone de convergence entre les objectifs personnels des salariés et les objectifs de l'entreprise. Lors de son élaboration, une négociation interindividuelle vise à impliquer chaque salarié dans la recherche d'un accord porté sur les objectifs d'amélioration et les contreparties en termes de moyens nécessaires ${ }^{3}$ à l'atteinte des objectifs et en termes de compléments de salaire. (Savall et Zardet, 1987).

Par ailleurs, elle implique le supérieur hiérarchique direct dans la présentation et l'explicitation des objectifs et, lui donne un pouvoir de décision sur les moyens correspondants qu'il propose.

\footnotetext{
2 Nous utiliserons l'abréviation CAPN pour désigner le «contrat d'activité périodiquement négociable »

${ }^{3}$ Les moyens nécessaires à l'atteinte des objectifs : formation, organisation, dispositifs d'information, budgets, moyens matériels, etc. Cf. Savall S., \& Zardet V., Maîtriser les coûts et les performances cachés, p33, $6^{\text {ème }}$ édition, 2015, ECONOMICA, $1^{\text {ère }}$ édition 1989.
} 
S'appuyant sur les théories de la motivation exposées dans la partie précédente, la négociation interindividuelle vise à rendre visible une justice organisationnelle, puisque les récompenses sont considérées comme adaptées par les salariés qui les ont négociées. Elle augmente leur autonomie, ce qui leur donne le sentiment d'être des responsables, élément également considéré clé par ces théories.

\section{2. - Méthodologie de la recherche}

Notre recherche vise à développer simultanément des connaissances utiles pour les entreprises et pour l'enrichissement de la littérature, notre méthodologie s'inscrit dans le cadre de la rechercheintervention socio-économique de l'approche qualimétrique qui repose sur trois principes épistémologiques: l'interactivité cognitive, l'intersubjectivité contradictoire et la contingence générique (Savall et Zardet, 2004 ; Savall 2018). Afin de tester l'effet motivationnel de la négociation interindividuelle, en mesurant l'évolution de la motivation des salariés chinois à participer à un effort d'amélioration continue, nous avons établi trois paramètres de mesure de la motivation des salariés chinois à l'amélioration continue : (1) Paramètre qualitatif (TCR) Type de comportement nécessaire à la mise en œuvre d'une amélioration que le salarié refuse d'adopter ; (2) Paramètre quantitatif - (NTCR) Nombre de salariés qui refusent d'adopter le même type de comportement propice à la mise en œuvre d'une amélioration ; (3) Paramètre financier - (CEDC) Conséquence économique des dysfonctionnements dûs à ces différents TCR mais corrigibles.

\subsection{Terrain d'expérimentation}

Notre expérimentation a été effectuée dans une entreprise spécialisée dans la transformation de pièces de structure d'avions civils. Elle est située à Tianjin. L'entreprise pratique le LEAN depuis plusieurs années. La direction cherche à améliorer la performance économique de l'entreprise en s'appuyant sur une double approche, descendante lorsqu'elle implique les managers dans des activités telles que le chronométrage des opérations, la rationalisation des processus en utilisant le rapport $\mathrm{A} 3$, la mise en œuvre des solutions d'amélioration, mais aussi ascendante, lorsqu'elle pousse chaque salarié à proposer des idées d'amélioration. L'entreprise octroie des primes collectives liées au résultat productif, des primes individuelles et/ou collectives selon les 
idées d'amélioration. Notons que les idées suggérées n'apportent pas que des bénéfices à leurs auteurs, ils sont, en effet, considérés comme responsables des dégâts relatifs à leur mise en application. Ces deux démarches n'ont pas empêché un arrêt de l'amélioration continue de la performance économique que l'entreprise pensait retirer de son LEAN management. Nous avons eu accès à cette entreprise, en tant qu'intervenant-chercheur. Notre mission était d'aider la direction à relancer l'amélioration continue.

\subsection{Processus de notre recherche}

Le processus de notre recherche est composé de trois étapes :

(1) Entretiens diagnostics semi-directifs (4 managers, 10 ouvriers). Les entretiens portaient sur les dysfonctionnements perçus par chacun comme étant fréquents, mais corrigibles. Les interviewés ont été aussi invités, avec une grille d'entretien construite sur la base de travaux portant sur l'amélioration continue (cf. Annexe1), à identifier le TCR et à en expliquer les causes.

(2) Étude comparative entre un cas d'expérimentation de négociations interindividuelles et un cas contraste impliquant les mêmes salariés. Concrètement, nous avons joué un rôle de médiateur dans le cas expérimental, afin d'établir et de finaliser la négociation interindividuelle entre chaque salarié impliqué et son supérieur. Nous avons discuté avec chaque salarié des améliorations qu'il envisageait de réaliser et des récompenses qu'il souhaitait obtenir. Nous sommes revenus vers le supérieur, puis les salariés, et nous avons défini les conditions dans lesquelles un accord serait acceptable par les deux parties.

(3) Entretiens semi-directifs d'évaluation post expérimentation. Ces entretiens portaient sur les dysfonctionnements que chaque salarié a pu résoudre, ainsi que sa perception de la pertinence de la négociation interindividuelle.

Lors de chaque entretien, nous avons procédé à la prise manuelle de notes exhaustives, afin d'établir des phrases témoins. Nous avons dû procéder à la traduction, du chinois au français, de ces phrases témoins pour leur restitution auprès des chercheurs français.

\subsection{Traitement des données}


NÉGOCIATION INTERINDIVIDUELLE \& CONTEXTE SOCIO-POLITIQUE CHINOIS

Notre traitement des données qualitatives s'appuie sur la méthode proposée par Strauss et Corbin qui implique trois étapes de codage : codages ouvert, axial, et sélectif (Strauss et Corbin, 1998). Notre codage ouvert consiste en une étude mot à mot des phrases témoins. Nous attribuons un code commun aux mots qui ont le même sens et qui sont liés à notre sujet de recherche. Dans la phase du codage axial, nous regroupons les codes qui ont des sens liés les uns aux autres afin de construire une idée clé. Le regroupement des idées clés qui décrivent un même phénomène et ses causes permet de générer un soussous-thème. Nous conceptualisons ensuite de la même façon les sousthèmes. Enfin, les sous-thèmes sont ensuite regroupés par thèmes. Ceci nous permet d'identifier les TCR et d'en identifier les causes.

Nos données quantitatives incluent le nombre de salariés qui refusent de s'engager dans une amélioration (NTCR). L'évaluation du NTCR repose alors sur un calcul cumulatif de la quantité de salariés qui ont présenté le même TCR.

Suivant la méthode d'évaluation des coûts cachés de l'analyse socio-économique (Savall, 1979 ; Savall et Zardet, 1987), nos données financières mesurent la CEDC (conséquence économique des dysfonctionnements corrigibles qui se manifestent fréquemment à cause des différents TCR). Cette mesure combine : le temps alloué à la régulation des dysfonctionnements (TRD) et la contribution horaire moyenne à la valeur ajoutée sur coût variable (CHVACV). Le calcul de la CEDC correspond alors à la formule : $\mathrm{CEDC}=\mathrm{TRD} * \mathrm{CHVACV}$. Dans notre étude, le traitement du TRD inclue trois types d'informations collectées auprès des interviewés : la fréquence du dysfonctionnement (FD), le TRD personnel (TRDP) et le nombre de salariés concernés (N) (cf. Annexe 2). Le calcul du TRD correspond alors à la formule : $\mathrm{TRD}=\mathrm{FD} * \sum_{k=1}^{n} X_{k}$. En raison du non-accès à des données comptables complètes, nous faisons l'hypothèse que, étant donné que le salarié travaille 8 heures par jour et 264 jours par an, la CHVACV du salarié de l'entreprise E égale la valeur ajoutée créée par chaque salarié industriel en Chine en moyenne sur l'année 2016, soit 63 yuans/personne/heure. Le calcul de la CEDC correspond alors à la formule : $\mathrm{CEDC}=63 * \mathrm{TRD}$.

\section{3. - Résultats de la recherche}


La négociation interindividuelle aboutit à une véritable augmentation de la motivation des salariés chinois à l'amélioration continue, et ainsi, à une amélioration de la performance économique des entreprises considérées. Cela se perçoit dans l'évolution des comportements des salariés du groupe expérimental (cf. tableau 1). Parallèlement, cette évolution comportementale conduit à des actions d'amélioration menées par ces salariés : 4 dysfonctionnements fréquents ont été corrigés. Ce sont 174,41 yuans par jour de coûts cachés qui sont ainsi recyclés (cf. tableau 2). En revanche, on n'observe pas de nouveau comportement d'amélioration, ni d'évolution de motivation des ouvriers du groupe contraste. Ces résultats nous paraissent vérifier la pertinence de la négociation interindividuelle. L'évolution de la motivation des salariés du groupe expérimental semble liée à trois facteurs que nous développons dans les points suivants.

\subsection{Rétablissement de la justice organisationnelle au sein de l'entreprise}

La perception de l'équité entre la contribution et la rétribution est le facteur majeur qui mobilise les salariés (managers et ouvriers) chinois dans l'amélioration continue. De manière générale, les salariés prennent en comptes trois variables lorsqu'ils évaluent leur contribution : le temps et l'effort qu'ils y consacrent, le niveau et la durée des primes gagnées, les risques perçus quant à leur emploi.

"Comme je viens d'expliquer, ça ne permet pas de gagner quoi que ce soit et je prends des risques, rien ne peut me motiver à le faire... " (Manager 3)

"Le temps et l'énergie que j'ai investis dans cette affaire doivent être récompensés. Mais quand je m'implique, ça veut dire que j'accepte les risques, je peux être viré si nos performances ont tellement augmenté que l'entreprise a intérêt à virer quelqu'un. Je veux dire que le fait que j'accepte ce risque doit également être récompensé... »(Ouvrier 9)

"Par exemple, si ma solution permet à l'entreprise de gagner 10.000 yuans en plus chaque mois, elle doit me donner alors peut-être 100 yuans en plus chaque mois, ou un poste de gestionnaire... » (Ouvrier 13) 
NÉGOCIATION INTERINDIVIDUELLE \&

CONTEXTE SOCIO-POLITIQUE CHINOIS

\begin{tabular}{|l|c|c|}
\hline \multirow{2}{*}{ TCR évoqués par les managers du groupe expérimental } & \multicolumn{2}{c|}{$\begin{array}{c}\text { Nombre de managers concernés } \\
\text { (/4) }\end{array}$} \\
\cline { 2 - 3 } & Période N & Période N+1 \\
\hline Briser les barrières de statut bureaucratique & 1 & 1 \\
\hline Signaler les problèmes au supérieur & 1 & 0 \\
\hline Écrire et réformer les instructions du travail & 2 & 0 \\
\hline Aider les subordonnées à formuler leur suggestion & 1 & 1 \\
\hline Recueillir des données sur le terrain & 1 & 1 \\
\hline Briser les barrières fonctionnelles & 2 & 0 \\
\hline Aider les subordonnés à traiter les problèmes & 3 & 0 \\
\hline Transmettre les expériences et le savoir-faire & Nombre d'ouvriers concernés \\
\hline \multicolumn{1}{|c|}{ TCR évoqués par les ouvriers du groupe expérimental } & & $(/ \mathbf{5})$ \\
\cline { 2 - 3 } & Période N & Période N+1 \\
\hline Comprendre que l'amélioration continue fait partie intégrante du travail & 1 & 0 \\
\hline Focaliser sur les améliorations adaptées aux objectifs de l'entreprise et du \\
département & 4 & 4 \\
\hline Écrire et réformer les instructions du travail & 4 & 0 \\
\hline Signaler les problèmes au supérieur & 1 & 0 \\
\hline Être autonome dans l'application des solutions & 2 & 0 \\
\hline Présenter la logique des solutions & 2 & 0 \\
\hline Transmettre les expériences et le savoir-faire & 1 & 0 \\
\hline
\end{tabular}

Tableau 1 : Évolution des types de comportements nécessaires à la mise en œuvre de l'amélioration continue que les salariés refusent d'adopter (Tang, 2020)

\begin{tabular}{|c|c|c|c|c|c|c|c|}
\hline \multicolumn{2}{|c|}{$\begin{array}{l}\text { Dysfonctionnements } \\
\text { Conséquences } \\
\text { Économiques } \\
\text { (Yuans/jour) }\end{array}$} & \multirow{2}{*}{$\begin{array}{c}\begin{array}{c}\text { Outillages de } \\
\text { fixation } \\
\text { inadaptés à } \\
\text { l'usinage des } \\
\text { nouvelles } \\
\text { pièces }\end{array} \\
141.75\end{array}$} & \multirow{2}{*}{$\begin{array}{c}\begin{array}{c}\text { Manque de } \\
\text { mesures } \\
\text { propices à la } \\
\text { disposition des } \\
\text { outillages de } \\
\text { fixation }\end{array} \\
42\end{array}$} & \multirow{2}{*}{$\begin{array}{c}\begin{array}{c}\text { Retard dans la } \\
\text { détection de la } \\
\text { production } \\
\text { des pièces } \\
\text { défectueuses }\end{array} \\
\mathbf{6 1 4 . 2 5}\end{array}$} & \multirow{2}{*}{$\begin{array}{c}\begin{array}{c}\text { Manque de } \\
\text { mise à jour } \\
\text { des }\end{array} \\
\text { instructions } \\
\text { du travail }\end{array}$} & \multirow{2}{*}{$\begin{array}{c}\begin{array}{c}\text { Disposition } \\
\text { des outils } \\
\text { inadaptée à la } \\
\text { production }\end{array} \\
56.7\end{array}$} & \multirow{2}{*}{$\begin{array}{l}\text { Total : } \\
883.35\end{array}$} \\
\hline \multirow{3}{*}{ 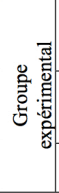 } & $\begin{array}{l}\text { Montant du } \\
\text { coût caché en } \\
\text { Période N }\end{array}$ & & & & & & \\
\hline & $\begin{array}{l}\text { Montant du } \\
\text { coût caché en } \\
\text { Période } \mathrm{N}+1\end{array}$ & 47.25 & 0 & 614.25 & 19.09 & 28.35 & 708.94 \\
\hline & $\begin{array}{l}\text { Amélioration } \\
\text { réalisée }\end{array}$ & 94.5 & 42 & 0 & 9.65 & 28.35 & 174.41 \\
\hline \multirow{3}{*}{ 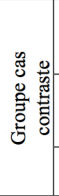 } & $\begin{array}{l}\text { Montant du } \\
\text { coût caché en } \\
\text { Période N }\end{array}$ & 141.75 & 42 & 614.25 & 28.65 & 56.7 & 883.35 \\
\hline & $\begin{array}{l}\text { Montant du } \\
\text { coût caché en } \\
\text { Période N }+1\end{array}$ & 141.75 & 42 & 614.25 & 28.65 & 56.7 & 883.35 \\
\hline & $\begin{array}{l}\text { Amélioration } \\
\text { réalisée }\end{array}$ & 0 & 0 & 0 & 0 & 0 & 0 \\
\hline
\end{tabular}

Tableau 2 : Conséquence économique de l'évolution de la motivation des salariés à l'amélioration continue (Tang, 2020) 
Concernant les risques, les salariés chinois soulignent tous qu'une amélioration apportée à la productivité provoque généralement une réduction du personnel. La spécialisation sur une tâche unique limite le développement des compétences polyvalentes des salariés chinois, ainsi que leurs perspectives d'embauche future. Cependant les tâches fortement standardisées et simplifiées réduisent considérablement l'exigence de compétence. Les ouvriers craignent d'être remplacés par une main d'œuvre moins qualifiée et à faible coût. Les managers craignent d'être remplacés par les ouvriers qualifiés. Les ouvriers et les managers refusent alors d'effectuer des améliorations qui nécessiteraient une transmission de savoir-faire. Ils réduisent ainsi la concurrence interne et externe à l'entreprise. Ils suivent en cela un schéma défensif largement décrit par Crozier (1964) sur un exemple français déjà bien ancien.

"Tous sont bien standardisés...Le savoir-faire me permettant de faire ce travail est facile à apprendre... » (Manager 4)

"Parce que ce n'est pas difficile pour les ouvriers d'avoir accès à des connaissances permettant de faire mon travail..." (Manager 3)

"C'est courant que les entreprises remplacent les ouvriers urbains par des ouvriers paysans...je suis bien conscient que mes compétences ne me permettent pas d'être embauché pour d'autres types de travail... » (Ouvrier 14)

Les salariés chinois estiment donc que le développement de leur motivation à l'amélioration continue doit impliquer des récompenses pertinentes en fonction de l'effort, de leur apport spécifique à l'amélioration et du risque qu'ils courent. La perception de l'équité reste subjective et personnelle. Il est difficile d'évaluer de manière objective l'effort que chaque salarié déploie dans l'amélioration continue et le risque qu'il court. La négociation interindividuelle peut donc être considérée comme pertinente, dans la mesure où elle est susceptible d'arriver à un équilibre entre contribution et rétribution mutuellement acceptable.

"Ce qui me motive, c'est le fait que je puisse dire à mon supérieur, grâce à la négociation, ce que je souhaite en matière de récompenses pour mon implication dans l'assistance aux ouvrier (en donnant des suggestions d'amélioration susceptibles d'être acceptées) ... » (Manager 3) 
NÉGOCIATION INTERINDIVIDUELLE \&

CONTEXTE SOCIO-POLITIQUE CHINOIS

"Pendant la négociation, j'ai demandé à mon directeur une somme d'argent en tenant compte de l'apport de ma proposition et des efforts que j'allais devoir déployer...j'ai l'impression que ma rémunération est plus juste qu'avant... »(Ouvrier 5)

Parallèlement, la négociation interindividuelle permet au salarié de définir avec son supérieur les critères d'une amélioration réussie et les conditions de l'attribution des récompenses. Cela contribue à passer d'une gestion autocratique à une gestion davantage transparente et participative. L'implication dans le processus de prise de décision est un puissant facteur de motivation.

"Ce que j'ai demandé reste très raisonnable...Mais c'est plutôt le fait qu'on me laisse définir les conditions dans lesquelles je peux obtenir cette somme d'argent qui me motive... » (Manager 4)

"C'est moi qui décide quelle chose doit être faite, à quel moment et de quelle façon...ça me donne l'impression que les récompenses sont attribuées de manière plus équitable lorsque je suis impliqué dans la définition, avec mon chef, des mesures pour lesquelles je vais toucher cette somme d'argent..." (Ouvrier 5)

\subsection{Développement de la motivation des ouvriers à travers l'autonomie du travail et la responsabilisation}

Au sein de la société chinoise actuelle, les ouvriers constituent une classe marginale. Leur métier est souvent considéré comme étant dévalorisant car il requiert un faible niveau de compétences, et apporte un faible niveau de revenus. Ce mépris de la société pour les ouvriers est intériorisé par eux dans un manque d'estime de soi.

"Comme nous gagnons peu et que nous sommes souvent considérés comme peu compétents, dans notre vie privée, personne n'a d'estime pour nous... » (Ouvrier 5)

"Dans cette société, on te respecte lorsqu'on suppose que tu es riche ou que tu as la capacité de gagner beaucoup d'argent. C'est pour ça que personne ne respecte les ouvriers..." (Ouvrier 10)

"Le travail que je fais chaque jour consiste uniquement en ça, c'est simple comme tout. En plus c'est super standardisé, On n'a 
pas besoin d'être compétent pour le faire... Mais l'entreprise vise à standardiser encore nos opérations lorsqu'elle travaille sur les instructions de travail, ça réduit encore nos marges... » (Ouvrier 5)

"Le LEAN impose des contraintes sur nos opérations. Nous ne pouvons pas travailler avec nos propres méthodes et somme obligés de suivre les instructions... »(Ouvrier 12)

Si la démotivation des ouvriers chinois se doit beaucoup à la dévalorisation du travail, elle est aussi imputée à une mauvaise réaction des managers. Les opinions des ouvriers sont rarement sollicitées par les managers autant pour la production que pour l'amélioration continue. Le manque de feed-back et de soutien des managers entraîne chez les ouvriers le sentiment d'être méprisés et réduit leur implication dans l'amélioration continue.

" J'ai l'impression que mon implication n'a aucune importance pour les managers...Parce que s'ils veulent que je sois impliqué dans mon travail, ou dans l'amélioration, ils doivent au moins venir me voir et me demander ce que j'en pense... » (Ouvrier 2) "Lorsqu'on leur raconte des idées, ils ne donnent jamais leur avis...ça sert à quoi mon implication dans ce cas-là..." (Ouvrier 3)

"C'est pareil dans cette entreprise, ce sont les managers qui nous dirigent. Ils n'ont pas d'estime pour nous... » (Ouvrier 10)

Dans ce contexte, la contribution de la négociation interindividuelle à la motivation des ouvriers est évidente. Il s'agit d'abord d'un enrichissement du travail des ouvriers chinois. En effet, l'élaboration de la négociation interindividuelle implique chaque salarié dans la conception du travail même, à travers la définition avec son supérieur des objectifs d'amélioration, des activités futures et des mesures de contrôles correspondants.

"Cette négociation me permet de définir avec mon chef des choses telles que ma solution, la façon dont je vais la mettre en cuvre, le délai etc. Cela permet donc de développer mon autonomie dans la prise de décision... » (Ouvrier 8)

Comparée au travail d'exécution strictement contrôlé par les managers supérieurs et aux pratiques chinoises habituelles du LEAN, l'élaboration de la négociation interindividuelle ouvre de nouvelles 
NÉGOCIATION INTERINDIVIDUELLE \& CONTEXTE SOCIO-POLITIQUE CHINOIS

possibilités d'autoréalisation aux ouvriers chinois. Il y a bien une augmentation de l'estime de soi qui ne peut que rejaillir sur la motivation au travail.

"Comme c'est moi qui ai décidé de ce que je dois mettre en ouvre et du délai, je peux alors autocontrôler mes actions, donc je suis responsable de ce que je fais... » (Ouvrier 6)

"Je peux non seulement proposer ce que j'aurai à faire, mais aussi la façon que de le faire, les bénéfices escomptés, etc... Donc, c'est moi qui suis responsable de la mise en æuvre et du contrôle de mes actions... " (Ouvrier 7)

\subsection{Augmentation de l'expectation des salariés à travers la co- définition des objectifs}

L'expectation renvoie à la perception du salarié de la probabilité d'atteindre un objectif donné lorsqu'il déploie des efforts en ce sens. Elle repose sur des facteurs personnels (compétence, aptitude, expérience, confiance en soi, etc.) et sur la difficulté perçue à atteindre l'objectif. Il est alors possible d'augmenter l'expectation des salariés par une prise en compte de ces facteurs personnels et la fixation d'objectifs adaptés. L'ouvrier ne sait pas à l'avance s'il peut atteindre des objectifs fixés par d'autres que lui, par contre, s'il participe à la définition de ces objectifs, alors il peut être assuré de les atteindre.

"Ce que j'ai négocié avec mon chef reste tout à fait dans le cadre de ma capacité, je suis alors $100 \%$ sûr que je peux y arriver... » (Ouvrier 7)

"Ce que j'ai signalé à mon chef est un problème pour lequel j'ai déjà la solution, je suis sûr de pouvoir résoudre ce problème et toucher la récompense... »(Ouvriers 6)

Parallèlement, le manque de soutien entre managers et ouvriers complique l'atteinte de leurs objectifs d'amélioration. En effet, les habitudes de management chinois ne favorisent pas la coopération verticale au sein des entreprises. Pour les managers, l'atteinte d'un objectif d'amélioration dépend non seulement des efforts de leur part, mais aussi du respect de leurs instructions par les ouvriers. La résistance de ces derniers est peu visible mais efficace.

" J'ai l'impression que c'est vraiment difficile de faire ça à mon niveau. Parce que j'ai essayé plusieurs fois d'accroitre la productivité chez nous, mais comme les ouvriers ne respectent 
jamais les consignes, du coup, à chaque fois, ça a été un échec... » (Manager 2)

"C'est toujours sur la productivité! L'entreprise cherche toujours à augmenter la production ou à réduire le nombre d'ouvriers... » (Ouvrier 7)

"Quand les managers viennent évaluer notre productivité, nous faisons exprès de ralentir au maximum notre rythme de production pour maintenir au niveau minimum la production... » (Ouvrier 14)

De leur côté, les managers sont réticents à fournir aux ouvriers certaines informations techniques permettant d'atteindre les objectifs fixés, mais qui rendraient les ouvriers trop compétents à leurs yeux. Cela constitue aussi un frein à l'atteinte des objectifs par les ouvriers et une limite à leur implication.

"Sur ce problème, les managers n'ont jamais demandé mon avis. En fait, j'ai des idées, mais comme ils ne m'expliquent jamais des points techniques, je ne peux le faire même si j'ai envie... » (Ouvrier 8)

La négociation interindividuelle oblige managers et ouvriers à réduire ces freins, mais elle présente des avantages pour les deux catégories. Pour les cadres, elle leur donne une meilleure visibilité sur les progrès à venir de l'organisation, ainsi que sur les changements susceptibles d'être mis en œuvre. Pour les ouvriers, elle constitue une reconnaissance et une ouverture vers des tâches susceptibles de faire évoluer leurs compétences, leur ouvrant l'accès à d'autres emplois. L'avantage économique pour l'entreprise est évident, puisqu'elle permet un retour à une démarche d'amélioration continue.

"Comme ce sont eux qui m'ont proposé cette solution d'amélioration...Pour moi, ce sont alors des marges d'action qu'ils sont prêts à réduire. J'ai tenu compte de tout ça lorsque j'ai retravaillé les processus. Pour eux, c'est plus facile de respecter ces processus améliorés... » (Manager 2)

" J'ai signalé à mon chef que j'ai juste besoin qu'on m'explique quelques points techniques. Le manager $X$ est venu me voir quelques jours plus tard... Une fois que j'ai bien compris, c'est devenu facile pour moi, j'ai pu tester immédiatement ma solution... »(Ouvrier 8) 
NÉGOCIATION INTERINDIVIDUELLE \& CONTEXTE SOCIO-POLITIQUE CHINOIS

Les résultats de notre recherche révèlent la pertinence de la négociation interindividuelle dans le développement de la motivation des salariés à la recherche d'amélioration continue.

\section{4. - Discussion et conclusion}

Comme le montrent les résultats de notre recherche, les managers et les ouvriers sont très exigeants quant au rapport entre efforts et récompenses. Comme chaque catégorie de salariés a ses propres préférences en terme de récompense, les facteurs permettant de stimuler la motivation des managers ne sont pas identiques à ceux des ouvriers. Les managers sont soucieux de leur autorité au sein l'entreprise. Pour les ouvriers, c'est l'absence d'opportunités de progression de carrière qui nourrit leur désaffection vis-à-vis du travail. On le voit, cela nécessiterait une quasi-personnalisation de la GRH. Malheureusement, l'entreprise chinoise aura beaucoup de mal à décentraliser les décisions de GRH pour permettre cette personnalisation. Cependant, notre recherche montre que l'application de négociations interindividuelles peut aider une entreprise chinoise à mettre en œuvre des pratiques de GRH personnalisées et à s'éloigner du paradigme de la soumission. De manière concrète, l'application de la négociation interindividuelle permet au salarié d'exprimer ses besoins et/ou ses inquiétudes. Elle permet à l'entreprise de mémoriser les préférences de chaque salarié. Ceci peut être considéré comme un nouveau moyen de créer un sentiment d'équité chez les salariés.

Les résultats de notre recherche montrent également que les salariés chinois sont conscients d'impacts négatifs de l'application du LEAN management renforcée par le paradigme de la soumission: stress au travail, réduction de l'autonomie au travail, limitation des compétences individuelles, etc. Dans un contexte dans lequel la sécurité de l'emploi n'est pas garantie par l'entreprise, ces impacts négatifs du LEAN management conduisent les salariés à adopter un comportement de réserve, voire de dissidence, face à des propositions d'amélioration susceptibles d'éliminer leurs marges d'action. Les salariés chinois de la génération actuelle sont davantage exigeants quant aux conditions de travail. Parallèlement, ils agissent d'autant plus volontairement qu'ils ont l'espoir d'une récompense valant l'effort à fournir. Il ne nous apparaît possible d'établir un rapport équitable entre l'effort et la 
récompense qu'à travers la négociation interindividuelle. Par ailleurs, nous nous devons de souligner que la négociation interindividuelle, telle qu'utilisée dans l'approche socio-économique, est compatible avec le LEAN. Comme le montrent les résultats de recherche, la mise en œuvre de la négociation interindividuelle offre à l'entreprise praticienne du LEAN la possibilité de stimuler la motivation des salariés à s'engager dans l'amélioration continue. De plus, la mise en œuvre de négociations interindividuelles contribue à rendre motivant le système sanctions-récompenses. Elle doit donc être considérée comme étant une démarche permettant d'améliorer la performance sociale de l'entreprise.

Notre dernier point porte sur l'aspect « invisible » de la négociation. Il n'est pas évident de donner un pouvoir de négociation à un groupe de salariés dans le contexte socio-politique chinois actuel, car tout effort de représentation des volontés spécifiques d'un groupe de salariés risque d'être perçu comme une remise en cause, politiquement inacceptable, de la primauté du syndicat. Notre intervention au sein de l'entreprise montre que la mise en œuvre de négociations médiatisées par l'intervenant-chercheur peut être une première étape pour introduire un management plus participatif et mobilisateur. Dès que l'intervenant-chercheur s'est retiré, les cadres se sont avérés incapables d'assumer à eux seuls le maintien d'une négociation interindividuelle. Le maintien d'une négociation invisible peut donc, à ce stade, être considéré comme étant un moyen de garantir l'implémentation durable d'un management qui s'éloigne du dogme de la soumission. Sa généralisation nécessitera un changement d'attitude profond des managers et des chercheurs chinois. Nous pensons que le recours à des organisations externes est encore plus nécessaire ici que dans les pays occidentaux, en ce que le médiateur ne fait pas que de la médiation, il introduit aussi, subrepticement, un changement de pratiques managériales dont la Chine nous semble avoir particulièrement besoin.

Les résultats obtenus montrent à l'évidence que la négociation interindividuelle, issue du management socio-économique, permet une amélioration chiffrable substantielle des coûts cachés. De même, une amélioration visible de la motivation des cadres et des ouvriers concernés a bien été constatée. Si les cadres de cette usine n'ont pas souhaité aller plus à fond dans l'expérimentation, cela nous paraît relever davantage d'une peur de remettre en question leur propre statut que d'une crainte de déplaire aux autorités. Ces dernières ne se sont jamais manifestées sur cette expérimentation qu'elles ne pouvaient 
NÉGOCIATION INTERINDIVIDUELLE \& CONTEXTE SOCIO-POLITIQUE CHINOIS

cependant ignorer. Notre expérience des expérimentations chinoises nous permet d'affirmer que ce silence des autorités prouve qu'elles acceptent ce type de changement, dans la mesure où les aspects positifs dépassent les inquiétudes qu'elles pourraient soulever chez certains cadres du Parti. Si jamais, les autorités voulaient l'arrêt de ce type d'expériences, elles sauraient faire passer le message sans ambiguiité aucune. Nous pouvons donc conclure qu'effectivement la négociation interindividuelle est applicable en Chine, mais que sa mise en œuvre demande de procéder avec un doigté particulier.

\section{RÉFÉRENCES BIBLIOGRAPHIQUES}

ADAMS J S., « Toward and understanding of inequity », Journal of Abnormal and Social Psychology, vol 67, N5, 1963, p422.- 436.

BESSANT J., \& FRANCIS D., (1999), «Developing strategic continuous improvement capability », International Journal of Operations \& Production Management, vol 19, N¹1, 1999, p1106.- 1119.

BESSANT J., et al., "An evolutionary model of continuous improvement behavior », Technovation, vol 21, 2000, p67.-77.

Charles-Pauvers B., et al., Les déterminantes psychologiques de la performance du travail: un bilan des connaissances et proposions de voies de recherché, Note du LIRHE, 2006.

DeCI E., \& Ryan R., Intrinsic Motivation and Self-Determination in Human Behavior, Plenum Press, New York, 1985.

Emiliani L., " Continuous personal improvement », Journal of Workplace Learning, vol 10, $\mathrm{N}^{\circ} 1,1998$, p29.-38.

FOREST J F., «Rémunération au mérite et motivation au travail: perspectives théoriques et empiriques pour la fonction publique française », Revue Internationale des Sciences Administratives, vol 74, 2008, p345.-359.

Greeberg J., «A taxonomy of Organizational Justice Theories », Academy of Management Review, vol 12, $\mathrm{N}^{\circ} 1,1987, \mathrm{p} 9 .-22$.

Hackman J., \& Oldham G., « Work Redesign and Motivation », Professional Psychology, vol 11, №3, 1980, p445.-455.

Herzberg F., " One more time: how do you motivate employees », Harvard Business Review, Reprint 87507, September-October, 1987, p5.-16.

Imai M., (1986), Kainzen: The Key To Japan's Competitive Success, China Machine Press, Beijing, $1^{\text {ère }}$ édition 2012 version chinoise, 1 ère édition 1986. 
Locke A., \& LAtham G., Goal setting, a motivational technique that works! Englewood Cliffs, NJ: Prentice Hall, 1984.

LIKER K., The Toyota way: 14 management principles from the world's greatest manufacturer, McGraw-Hill, New York, 2004.

KANFER R., « Motivation theory and industrial and organizational psychology », in Dunnette D., \& Hough M., Handbook of industrial and organizational psychology, Palo Alto, Consulting Psychologists Press, vol 1, p75.-170.

$\mathrm{KocH}$ T., et al., «10 commandments for the boss of a company implementing LEAN philosophy », Management and Production Engineering Review, vol 3, N², 2012, p62.-78.

MASLOW A., «A theory of human motivation», Psychological Review, vol 50, $\mathrm{N}^{\circ} 4,1943, \mathrm{p} 370 .-396$

NAKANE J., \& HALL W., « Ohno’s Method: Creating a survival work culture », Target, vol 18, $\mathrm{N}^{\circ} 1,2002$, p6.-15.

Ohno T., Toyota Production System, Beyond Large-Scale Production, 1988 by Taylor \& Francis Group, LLC, English translation by Productivity Press, 1978.

Robinson G., \& Schroeder M., « The role of front-line ideas in Lean performance improvement », The Quality Management Journal, vol 16, $\mathrm{N}^{\circ} 4,2009$, p27.-40.

Rousseau D., "Psychological and implied contracts in organizations ", Employee Responsibilities and Rights Journal, N²2, 1989, p121.139.

RoulleAu-Berger L. (dir.), La nouvelle sociologie chinoise, CNRS éditions, Paris, 2008.

RUFFIER J., Faut-il avoir peur des usines chinoises? Compétitivité et pérennité de l'atelier du monde, L'Harmattan, Paris, 2006.

SAVAGEAU J., « World-class suggestion systems still work well », The Journal for Quality and Participation, vol 19, 1996, p86.-90.

SAVAll H., Enrichir le travail humain dans les entreprises et les organisations, Dunod, Études économiques, Paris, 1975.

SAVAll H., Reconstruire l'entreprise: Analyse socio-économique des conditions de travail, Dunod, Paris, 1979.

Savall H., Peron M., Zardet V., \& Bonnet M., Le Capitalisme Socialement Responsable Existe, Edition EMS, MANAGEMENT \& SOCIETE, 2015.

Savall H., \& Zardet V., Maîtriser les coûts et les performances cachés, Économica, Paris, 1987.

SAVAll H., \& ZARDET V., Recherche en sciences de gestion: approche qualimétrique - Observer l'objet complexe, Économica, Paris, 2004.

SAVAll H., \& ZARDET V., "Contribution de la théorie socio-économique des organisation à l'audit social », IAS, Université d'été, 2005, 1-2 septembre, Lille 
NÉGOCIATION INTERINDIVIDUELLE \&

CONTEXTE SOCIO-POLITIQUE CHINOIS

SAVAll H., \& ZARDET V., "Théorie socio-économique des organisations : impacts sur quelques concepts dominants dans les théories et pratiques managériales », Colloque et séminaire doctoral international ISEOR - Academy of Management, 2006, 24-25 Avril, Lyon

Savall H., Zardet V., Reconstruire l'entreprise: les fondements du management socio-économique, Dunod, Paris 2014.

Savall H., \& ZARDET V., « Reflecting on Conventional Wisdom: Learning From the Database » In Bruno F., \& Savall H., The Socio-Economic Approach to Management Revisited: The Evolving Nature of SEAM in the 21st Century, INFORMATION AGE PUBLISHIING, ING, Charlotte, NC, 2015, p29-53.

Spear S., \& Bowen H., « Decoding the DNA of the Toyota Production System ", Harvard Business Review September-October, 1999, p97.-106.

Strauss L., \& CORBIN M., Basics of Qualitative Research: Techniques and Procedures for Developing Grounded Theory, Sage Publications, Thousand Oask, 1998.

SuÁREZ-BARRAZA F., et al., « Thoughts on KAIZEN and its evolution: Three different perspectives and guiding principles », International Journal of Lean Six sigma, vol 2, n 4, 2011, p288.-308.

VRoom H., Work and Motivaiton, John Wiley \& Sonc Inc, New York, 1964

Womack J., \& Jones T., Lean Thinking: Banish Waste and Create Wealth in Your Corporation, Simon and Schuster, New-York, 1996.

ZHAO W., et Rigas A., « L'atelier du monde peut-il évoluer? Le cheminement des entreprises chinoises de l'émergence à l'innovation », Marché et Organisation, (2), 2014, p33.-55. 


\section{Annexe 1 : Nomenclature des comportements nécessaires pour la mise en ouvre d'une amélioration (TANG, 2017)}

\begin{tabular}{|c|c|}
\hline $\begin{array}{c}\text { Comportements nécessaires des salariés } \\
\text { (managers et ouvriers) } \\
\text { pour la mise en œuvre de l'amélioration continue }\end{array}$ & $\begin{array}{c}\text { Comportements nécessaires des } \\
\text { managers } \\
\text { pour la mise en œuvre de l'amélioration continue }\end{array}$ \\
\hline \multicolumn{2}{|c|}{ Étape 1 : Consolider la conscience } \\
\hline $\begin{array}{l}\text { - Comprendre que l'amélioration continue fait } \\
\text { partie intégrante du travail } \\
\text { - Focaliser sur les améliorations adaptées aux } \\
\text { objectifs de l'entreprise et du département } \\
\text { - Adopter l'esprit de «Do it yourself » et «Do it } \\
\text { rightnow » } \\
\text { - Penser aux clients internes }\end{array}$ & $\begin{array}{l}\text { - Briser les barrières de statut bureaucratique } \\
\text { - Harmoniser les messages d'amélioration } \\
\text { - Aider et soutenir les subordonnés } \\
\text { - Servir d'exemple aux subordonnés }\end{array}$ \\
\hline \multicolumn{2}{|c|}{ Étape 2: Identifier le problème } \\
\hline $\begin{array}{l}\text { - Écrire les instructions du travail } \\
\text { - Observer et identifier les gaspillages } \\
\text { - Examiner les processus et le manuel de travail } \\
\text { - Signaler les problèmes }\end{array}$ & $\begin{array}{c}\text { - Recueillir des données sur le terrain } \\
\text { - Écouter les subordonnés et réagir à leurs } \\
\text { observations/actions } \\
\text { - Aider les subordonnés à formuler des suggestions } \\
\text { - Aider les subordonnés à communiquer leurs } \\
\text { suggestions }\end{array}$ \\
\hline \multicolumn{2}{|c|}{ Étapes 3 et $4:$ Analyser le problème et identifier les causes } \\
\hline $\begin{array}{c}\text { - Ne pas blâmer les acteurs à l'origine du problème } \\
\text { - Utiliser des méthodes/outils formels } \\
\text { - Focaliser sur des améliorations prioritaires du } \\
\text { département puis de l'entreprise } \\
\text { - Briser les barrières fonctionnelles }\end{array}$ & $\begin{array}{c}\text { - Aider les subordonnés à traiter les problèmes } \\
\text { - Aider les subordonnés à formuler des suggestions } \\
\text { - Écouter les subordonnés et réagir à leurs } \\
\text { observations/actions }\end{array}$ \\
\hline \multicolumn{2}{|c|}{ Étape 5 : Projeter des solutions } \\
\hline $\begin{array}{c}\text { - Présenter la logique des solutions } \\
\text { - Évaluer la solution en fonction des objectifs de } \\
\text { l'entreprise et du département } \\
\text { - Préciser le délai d'application } \\
\end{array}$ & $\begin{array}{c}\text { - Aider les subordonnés à traiter les problèmes } \\
\text { - Écouter les subordonnés et réagir à leurs } \\
\text { observations/actions }\end{array}$ \\
\hline \multicolumn{2}{|c|}{ Étape 6: Appliquer les solutions } \\
\hline $\begin{array}{l}\text { - Etre autonôme dans l'application des solutions } \\
\text { - Modifier et reconstruire les équipements }\end{array}$ & $\begin{array}{l}\text { - Aider les subordonnés à traiter les problèmes } \\
\text { - Écouter les subordonnés et réagir à leurs } \\
\text { observations/actions }\end{array}$ \\
\hline \multicolumn{2}{|c|}{ Étape $7:$ Confirmer les résultats } \\
\hline $\begin{array}{c}\text { - Utiliser des méthodes/outils formels } \\
\text { - Évaluer les résultats en fonction des objectifs de } \\
\text { l'entreprise et du département }\end{array}$ & $\begin{array}{l}\text { - Vérifier les résultats avec les données du terrain } \\
\text { - Écouter les subordonnés et réagir à leurs } \\
\text { observations/actions } \\
\text { - Mettre en lumière la contribution de chacun }\end{array}$ \\
\hline \multicolumn{2}{|c|}{ Étape 8 : Normaliser } \\
\hline $\begin{array}{l}\text { - Réformer les instructions du travail et les } \\
\text { processus } \\
\text { - Transmettre les expériences et le savoir-faire }\end{array}$ & $\begin{array}{c}\text { - Soutenir l'apprentissage des personnels } \\
\text { - Écouter les subordonnés et réagir à leurs } \\
\text { observations/actions } \\
\text { - Mettre en lumière la contribution de chacun }\end{array}$ \\
\hline \multicolumn{2}{|c|}{$\begin{array}{l}\text { Source : Bessant \& Francis (1999); Bessant et al, (2000); Emiliani (1998); Imai (1986); Koch et al, (2012); } \\
\text { Liker (2004); Nakane \& Hall (2002); Ohno 1(978); Robinson \& Schroeder (2009); Savageau (1996); } \\
\text { Spear \& Bowen (1999); Suárez-Barraza et al, (2012) }\end{array}$} \\
\hline
\end{tabular}


NÉGOCIATION INTERINDIVIDUELLE \&

CONTEXTE SOCIO-POLITIQUE CHINOIS

\section{Annexe 2 : Exemple de calcul du TRD à partir des informations collectées auprès des interviewés}

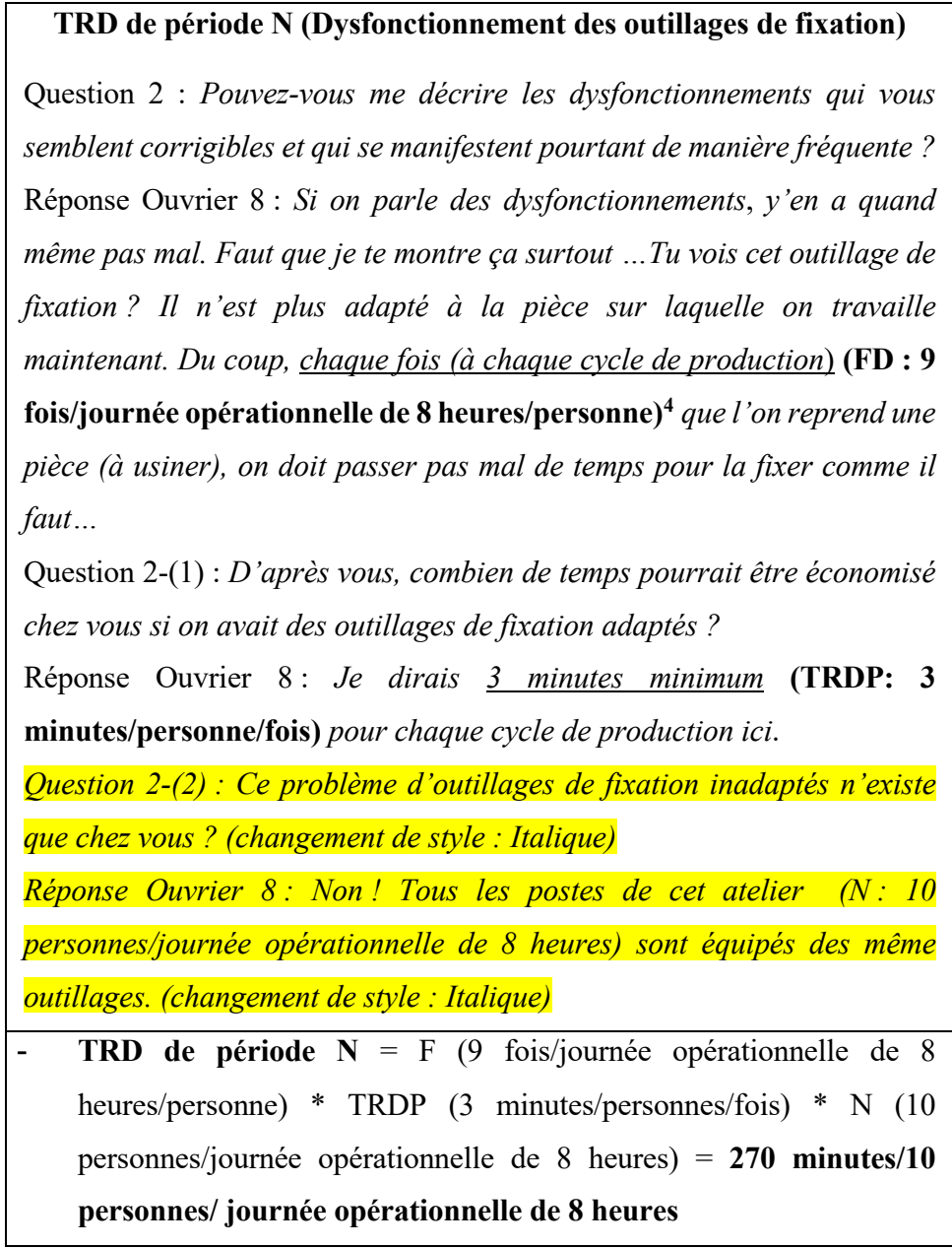

\footnotetext{
4 Temps de travail par jour $(8 \mathrm{~h} * 60 \mathrm{~min})-$ Temps de pause $(15 \mathrm{~min}+45 \mathrm{~min}+15$ min) /Cycle de production $(45 \mathrm{~min})$
} 
- Interprétation : Dans une journée opérationnelle de 8 heures, 10 salariés au sein de l'atelier production sont impactés par les outillages de fixation inadaptés et ont besoin de passer au total 270 minutes pour la régulation de ce dysfonctionnement. 